\title{
Plasmodium infection prevents recurrence and metastasis of hepatocellular carcinoma possibly via inhibition of the epithelial-mesenchymal transition
}

\author{
YUN LIANG ${ }^{1,2^{*}}$, XIAO CHEN ${ }^{2,3^{*}}, \mathrm{ZHU}^{\mathrm{TAO}}{ }^{4}, \mathrm{MENG} \mathrm{MA}^{2,5}, \mathrm{DICKSON}$ ADAH $^{2}, \mathrm{XIAOFEN} \mathrm{LI}^{2}, \mathrm{LINGLING} \mathrm{DAI}^{2}$, \\ WENTING DING $^{4}$, SONGWE FANUEL ${ }^{2}$, SITING ZHAO ${ }^{2}$, LI QIN ${ }^{4}$, XIAOPING CHEN $^{2}$ and XIAOWEN ZHANG ${ }^{6}$ \\ ${ }^{1}$ Department of Hepatobiliary Surgery, The First Affiliated Hospital of Kunming Medical University, \\ Kunming, Yunnan 650032; ${ }^{2}$ State Key Laboratory of Respiratory Disease, Center of Infection and Immunity, \\ Guangzhou Institutes of Biomedicine and Health, Chinese Academy of Sciences, Guangzhou, Guangdong 510530; \\ ${ }^{3}$ Department of Medical Oncology, The First Affiliated Hospital of Kunming Medical University, Kunming, Yunnan 650032; \\ ${ }^{4}$ CAS-Lamvac Biotech Co., Ltd., Guangzhou, Guangdong 510670; ${ }^{5}$ The Ministry of Education Key Laboratory of \\ Laboratory Medical Diagnostics, College of Laboratory Medicine, Chongqing Medical University, Chongqing 400016; \\ ${ }^{6}$ Department of Hepatobiliary Surgery, The Second Affiliated Hospital of Kunming Medical University, \\ Kunming, Yunnan 650101, P.R. China
}

Received November 14, 2020; Accepted March 9, 2021

DOI: $10.3892 / \mathrm{mmr} .2021 .12057$

\begin{abstract}
Postoperative recurrence causes a high mortality rate among patients with hepatocellular carcinoma (HCC). The current study aimed to determine the effects of Plasmodium infection on HCC metastasis and recurrence. The antitumor effects of Plasmodium infection were determined using two murine orthotopic HCC models: The non-resection model and the resection model. Tumour tissues derived from tumour-bearing mice treated with or without Plasmodium infection were harvested 15 days post-tumour inoculation. The expression levels of biomarkers related to
\end{abstract}

Correspondence to: Professor Xiaoping Chen, State Key Laboratory of Respiratory Disease, Center of Infection and Immunity, Guangzhou Institutes of Biomedicine and Health, Chinese Academy of Sciences, 190 Kaiyuan Avenue, Huangpu, Guangzhou, Guangdong 510530, P.R. China

E-mail: chen_xiaoping@gibh.ac.cn

Professor Xiaowen Zhang, Department of Hepatobiliary Surgery, The Second Affiliated Hospital of Kunming Medical University, 374 Dianmian Avenue, Wu Hua, Kunming, Yunnan 650101, P.R. China

E-mail: zhangxiaowenlu@hotmail.com

*Contributed equally

Abbreviations: HCC, hepatocellular carcinoma; EMT, epithelial-mesenchymal transition; OS, overall survival; CCR10, CC-chemokine receptor 10; Py, Plasmodium yoelii 17XNL

Key words: Plasmodium, hepatocellular carcinoma, metastasis, EMT epithelial-mesenchymal transition (EMT) and molecules associated with CC-chemokine receptor 10 (CCR10)-mediated PI3K/Akt/GSK-3ß/Snail signalling were identified using reverse transcription-quantitative PCR and western blotting. The results demonstrated that Plasmodium infection significantly suppressed the progression, recurrence and metastasis of HCC in the two mouse models. The expression levels of E-cadherin were significantly higher in the Plasmodium-treated group compared with that in the control group, whereas the expression levels of Vimentin and Snail were significantly lower in the Plasmodium-treated group. Furthermore, Plasmodium infection inhibited the activation of Akt and GSK-3 $\beta$ in the tumour tissues by downregulating the expression levels of CCR10 and subsequently suppressing the accumulation of Snail, which may contribute to the suppression of EMT and the prevention of tumour recurrence and metastasis. In conclusion, the results of the present study demonstrated that Plasmodium infection inhibited the recurrence and metastasis and improved the prognosis of HCC by suppressing CCR10-mediated PI3K/Akt/GSK-3 $\beta /$ Snail signalling and preventing the EMT. These results may be important for the development of novel therapies for $\mathrm{HCC}$ recurrence and metastasis, especially for patients in the perioperative period.

\section{Introduction}

The annual incidence of liver cancer increased by $75 \%$ between 1990 and 2015 worldwide (1). Hepatocellular carcinoma (HCC) comprise $\sim 75-85 \%$ of liver cancers (2), which constitute a huge health burden, especially in Asia where $72 \%$ of cases occur (3). Surgical resection is one of the main treatment methods for HCC; however, it cannot prevent postoperative recurrence (4). The HCC recurrence rate at 2 years post-surgery is $61.6 \%$ according to the statistics provided 
by the World Health Organization (2018) (2). At present, the metastasis of liver cancer cells cannot be prevented by surgical resection, and there are a lack of effective drugs for the prevention and treatment of metastasis (5).

The epithelial-mesenchymal transition (EMT) induces cancer metastasis by promoting the separation and invasion of cancer cells from homologous cells (6). E-cadherin has been reported to maintain the tight connection between cells and prevent cell invasion and metastasis, and the decrease in the levels of E-cadherin is a common EMT biomarker (7). The upregulation of Vimentin and the transcription factor Snail are also commonly used EMT biomarkers $(8,9)$. Recent studies have emphasized the vital role of EMT in the aggressive progression of HCC (10-13), in this regard further efforts for identification of EMT regulatory mechanisms will facilitate the development of novel strategies to combat metastasis. Chemokines and chemokine receptors serve key roles in the invasion and metastasis of malignant tumours (14-16). CC-chemokine receptor 10 (CCR10) has been demonstrated to be significantly upregulated in HCC specimens compared with the normal liver specimens (10). Furthermore, PI3K/Akt/GSK-3 $\beta$ has been demonstrated to be a classical signalling pathway via which CCR10 activates phosphorylation of Akt (16).

Plasmodium is a genus of single-cell protozoa that causes malaria, which may enter hepatocytes for dormancy or reproduction and lead to cell rupture, release merozoites and invade red blood cells (17). Although Plasmodium stays in the liver and causes black colouring of the tissue due to the deposition of the Plasmodium pigment, it does not damage liver function (18). Our previous studies have demonstrated that Plasmodium infection inhibits tumour development and metastasis in a murine Lewis lung cancer model (19-24). However, although Plasmodium infection has been demonstrated to suppress HCC angiogenesis by decreasing the infiltration of tumour-associated macrophages and reducing their matrix metalloproteinase 9 levels (25), the effects of Plasmodium infection on HCC metastasis and recurrence remain unclear.

To gain further insight into the effects of Plasmodium infection on HCC metastasis and recurrence, the antitumor effects of Plasmodium infection using two murine orthotopic models were studied, with an emphasis on the regulation of Plasmodium infection on EMT of HCC. Here, it was demonstrated that Plasmodium infection prevented recurrence and metastasis of HCC potentially via CCR10-mediated PI3K/Akt/GSK-3 $\beta /$ Snail signalling.

\section{Materials and methods}

Ethics statement. Ethical approval was provided by Guangzhou Institute of Biomedicine and Health, Chinese Academy of Sciences (approval no. N2019014; Guangzhou, China). All animal experiments in this study were performed following the standard guidelines for the care of animals, which were approved by the Welfare Committee of the Center of Experimental Animals. According to the international regulations, the mice were euthanised when the tumour diameter reached $2 \mathrm{~cm}$. All efforts were made to minimise animal suffering.
Antibodies. Western blotting was performed using the following primary antibodies: GAPDH (cat. no. ab9385; Abcam), E-cadherin (cat. no. BS1098; Bioworld Technology, Inc.), Snail (cat. no. 3879S; Cell Signaling Technology, Inc.), PI3K (cat. no. ab151549; Abcam), phosphorylated (p)-PI3K p85 (Tyr458)/p55 (Tyr199) (cat. no. 4228S), Akt (cat. no. 9272S), p-Akt (Ser473) (cat. no. 4060S), GSK-3 $\beta$ (cat. no. 12456S), p-GSK-3 $\beta$ (Ser9(cat. no. 5558S) (all Cell Signaling Technology, Inc.) and CCR10 (cat. no. ab3904; Abcam).

Animals, cells and parasites. Female C57BL/6 mice (age, 6-8 weeks) purchased from Beijing Vital River Laboratory Animal Technology Co., Ltd. were bred in the Animal Center of Guangzhou Institute of Biomedicine and Health $(\mathrm{GIBH}$; Guangzhou, China) according to the Guide to the Care and Use of Laboratory Animal Committee of the Institute. Mice were housed under specific pathogen-free conditions with a 12-h light/dark cycle and were provided food and water ad libitum. The Hepa1-6 luciferase liver cancer cell line derived from C57BL/6 mice was obtained from The First Affiliated Hospital of Sun Yat-Sen University and cultured in Dulbecco's modified Eagle's medium (DMEM; Gibco; Thermo Fisher Scientific, Inc.) supplemented with $10 \%$ foetal bovine serum (FBS; Gibco; Thermo Fisher Scientific, Inc.), and was incubated in a humidified atmosphere with $5 \% \mathrm{CO}_{2}$ at $37^{\circ} \mathrm{C}$. The Plasmodium yoelii nonlethal strain (Py17XNL) was obtained from the Malaria Research and Reference Reagent Resource Center (MR4).

Animal models. Mice undergoing surgery were anaesthetised by intraperitoneal injection of $240 \mathrm{mg} / \mathrm{kg} 1.25 \%$ Avertin. Two animal models were established. The tumour volumes were calculated as follows: Volume $=\left(\right.$ length $\mathrm{x}$ width $\left.{ }^{2}\right) / 2$. Mice were euthanized by exsanguination under anaesthesia with $240 \mathrm{mg} / \mathrm{kg}$ Avertin.

Non-resection model. The non-resection mouse model was established by intrahepatic injection of $5 \times 10^{5}$ Hepa1-6 luciferase cells into the left liver lobe. Female C57BL/6 mice $(n=56)$ were randomly divided into two groups $(n=28$ mice/group). The control group (Hep) received 5x105 Hepa1-6 luciferase cells only. The mice in the Plasmodium-infected group $\left(\right.$ Hep + Py) were orthotopically implanted with $5 \times 10^{5}$ Hepa1-6 luciferase cells and intraperitoneally injected with $5 \times 10^{5}$ Plasmodium yoelii $17 \mathrm{XNL}$-parasitised erythrocytes. Subsequently, 26 mice ( $\mathrm{n}=13 \mathrm{mice} /$ group) were randomly selected for observation of survival, dynamics of parasitaemia and weight changes. The tumours in the remaining 30 mice ( $n=15$ mice/group) were harvested on day 15 post-inoculation for further experiments.

Resection model. To investigate the effects of Plasmodium infection on postoperative recurrence, the resection mouse model was established by an intrahepatic injection of tumour pieces and tumour resection on day 21 post-implantation. Tumours were derived from subcutaneous tumour-bearing mice, which had been subcutaneously injected with $5 \times 10^{5}$ Hepa1-6 luciferase cells. The tumours were harvested and cut into $1-\mathrm{mm}^{3}$ pieces on day 14 post-inoculation. The tumour pieces were implanted into the livers (left liver lobe) of 20 mice, 
and in vivo luciferase imaging was used to monitor the survival of the transplanted tumours once a week in the following days. The mice underwent resection on day 21 post-inoculation to completely remove the transplanted tumour mass and were randomly divided into two groups according to the inoculation with or without Plasmodium on day 3 post-surgery. The primary outcomes were death and intraperitoneal metastasis caused by HCC in both groups.

In vivo luciferase imaging. To evaluate tumour survival, the luminescence properties of luciferase were used to track HCC in vivo. Each mouse was intraperitoneally injected with $10 \mu \mathrm{l} / \mathrm{g}$ potassium fluorescein as a substrate. The IVIS Spectrum (PerkinElmer, Inc.) in vivo imaging system was used to monitor the fluorescence emitted by Hepa1- 6 luciferase at 10-20 min post-injection.

Reverse transcription-quantitative PCR (RT-qPCR). Total RNA was extracted from tumour tissues using TRIzol ${ }^{\circledR}$ reagent (cat. No. 15596018; Invitrogen; Thermo Fisher Scientific, Inc.) and reverse-transcribed $\left(37^{\circ} \mathrm{C}\right.$ for $15 \mathrm{~min}, 85^{\circ} \mathrm{C}$ for $5 \mathrm{sec}$ and $4^{\circ} \mathrm{C}$ hold) using a cDNA Synthesis kit (cat. No. RR047A; Takara Bio, Inc.). qPCR was performed using TB Green Premix Ex Taq (cat. no. RR820A; Takara Bio, Inc.) with CFX96 Touch PCR machine (Bio-Rad Laboratories, Inc.). The qPCR procedures were as follows: Predenaturation at $95^{\circ} \mathrm{C}$ for $30 \mathrm{sec}$; denaturation at $95^{\circ} \mathrm{C}$ for $5 \mathrm{sec}$, annealing and extension at $60^{\circ} \mathrm{C}$ for $30 \mathrm{sec}\left(39\right.$ cycles); denaturation at $95^{\circ} \mathrm{C}$ for $10 \mathrm{sec}$; and finally melt curve at $65-95^{\circ} \mathrm{C}$ for $5 \mathrm{sec}$. The mRNA levels of the genes of interest were normalised to those of $\beta$-actin. The primer sequences used were as follows: $\beta$-Actin forward, 5'-TCTGGCACCACACCTTCTAC-3' and reverse, 5'-TCA TCTTTTCACGGTTGGCCT-3'; Vimentin forward, 5'-CTG CGAGAGAAATTGCAGGAG-3' and reverse, 5'-CTTTCA TACTGCTGGCGCAC-3'; CCR10 forward, 5'-CAAGCCCAC AGAGCAGGTCTC-3' and reverse, 5'-GATCGGGTAGTT CGTCTGGC-3'. The $2^{-\Delta \Delta \mathrm{Cq}}$ method was used to quantify the relative changes in gene expression (26). Each sample was tested in at least three independent replicates.

Protein extraction and western blotting. Tissues were lysed in RIPA lysis buffer (cat. no. KGP702-100; Nanjing KeyGen Biotech Co., Ltd.) containing $1 \%$ phenylmethanesulphonyl fluoride (Beyotime Institute of Biotechnology) and phosphatase inhibitor cocktail (cat. no. HY-K0021; MedChem Express) on ice for $30 \mathrm{~min}$ and subsequently centrifuged at $16,000 \mathrm{x} \mathrm{g}$ for $15 \mathrm{~min}$ at $4^{\circ} \mathrm{C}$ to collect the supernatants. After protein determination using a BCA assay, protein samples containing $15-20 \mu \mathrm{g}$ protein were separated using $10 \%$ polyacrylamide gel electrophoresis (SurePAGE; cat. no. M00665; GenScript) and transferred to polyvinylidene difluoride membranes (cat. no. ISEQ00010; EMD Millipore). The membrane was probed with anti-E-cadherin (1:500), anti-Snaill $(1: 1,000)$, anti-p-PI3K (1:1,000), anti-PI3K (1:1,000), anti-p-Akt ${ }^{\text {Ser43 }}$ (1:500), anti-Akt (1:300), anti-p-GSK3 $\beta^{\text {Ser9 }}$ (1:500), anti-GSK3 $\beta$ (1:500) and anti- $\beta$-actin $(1: 2,000)$ antibodies at room temperature for $2 \mathrm{~h}$. Then, membranes were washed three times, $5 \mathrm{~min}$ each, with Tris-buffered saline with $0.1 \%$ Tween-20 (TBST). After incubation with HRP-conjugated Goat anti-Mouse IgG (cat. no. ab205719; Abcam) or HRP-conjugated Goat anti-Rabbit
IgG (cat. no. ab205718; Abcam) at room temperature for $1 \mathrm{~h}$ $(1: 5,000)$, membranes were washed four times, 5 min each, with TBST. Then, the bands were detected using ECL reagents (cat. no. WBULS0500; EMD Millipore). Densitometry of band signals was quantified using Quantity One software (version 4.6.6; Bio-Rad Laboratories, Inc.). Data are presented as the ratio of the target protein to GAPDH densitometry values.

Giemsa stain. Giemsa stain was performed for determination of the ratio of Plasmodium-infected RBC in the blood. A small drop of mouse tail blood was collected on a glass slide to make a thin layer of blood smear. Then, using a Pasteur pipette, the thin film was fixed by carefully dropping methanol onto the thin film. The slides were placed on a drying rack on a flat surface for $\sim 2$ min to let the blood film dry completely. Then, Giemsa working solution (cat. no. 48900; Sigma-Aldrich; Merck $\mathrm{KGaA}$ ) was poured slowly on the slide until the blood films were covered. After 40 min of staining, the stain was washed from the slides and then the slides were allowed to air-dry. Finally, the ratio of parasitaemia was calculated using the following formula: Parasitaemia $(\%)=($ the number of Plasmodium-infected RBC per 1,000 RBC/1,000) x100\%.

Statistical analysis. Statistical analysis was performed using GraphPad Prism 8.0 (GraphPad Software, Inc.) and the R software (version 3.6.3; http://www.R-project.org) (27). Data of normally distributed variables are presented as the mean \pm standard deviation. Categorical data are presented as frequency and percentages. An independent sample Student's t-test was used to compare the means of two groups. The Fisher's exact test was used to compare the rate of metastasis between two groups. Overall survival (OS) in the two groups was estimated using the Kaplan-Meier method, and the OS differences between the two groups were compared using the log-rank test. $\mathrm{P}<0.05$ was considered to indicate a statistically significant difference. All of above experiments were repeated at least twice and the results were similar.

\section{Results}

Plasmodium infection significantly inhibits HCC progression and metastasis in a non-resection mouse model. In this experiment, luciferase imaging results revealed that Hepa1-6 cells were successfully implanted into all mice, and the fluorescence signal was significantly lower in Hep + Py group compared with that in the Hep group on day 15-post inoculation (Fig. 1A). The peak of infection appeared on $\sim$ day 18 post-intraperitoneal injection of the parasite in both models; the highest Plasmodium density reached on average 55-60\% and decreased to $\sim 5 \%$ on day 24 (Figs. S1A and B and S2). There was no significant difference in body weight between the two groups during the course of Plasmodium infection (in the treated group), whereas the weight was not comparable between the two groups thereafter since the majority of the mice in the control group died (Fig. 1B). Notably, the tumour-bearing mice treated with Plasmodium exhibited a higher survival rate $(53.8 \%, 7 / 13)$ and a longer OS time (until to the end of the study) compared with that observed in the control group (median interval, 21 days) (Fig. 1C). The tumour volume on day 15 post-inoculation in the Hep + Py group 
A

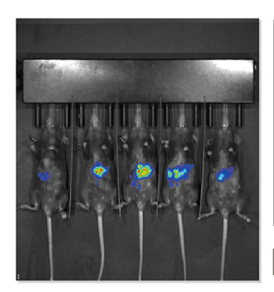

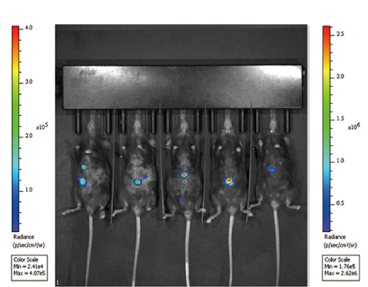

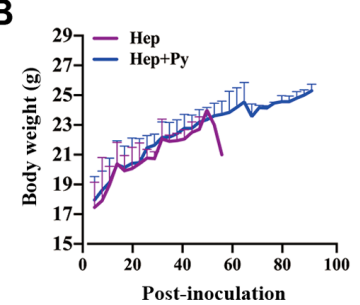

D
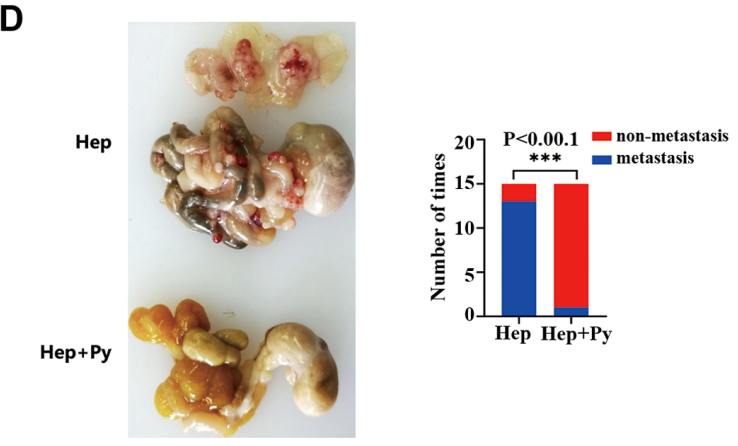

E

Hep

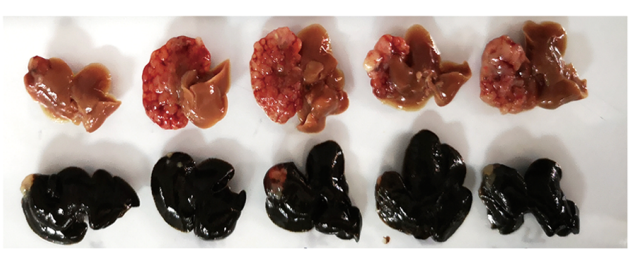

$\mathbf{F}$

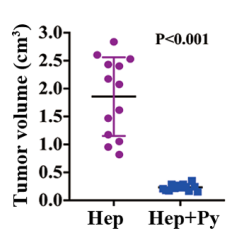

Hep+Py

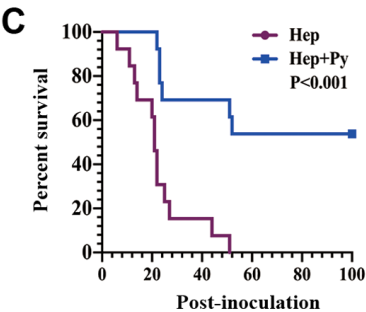

Figure 1. Plasmodium infection significantly inhibits HCC progression and metastasis in the non-resection mouse model. (A) Hepa1-6 cells were successfully implanted into the mice, which was confirmed by in vivo fluorescence detection. (B) Body weight curves of mice in the Hep and Hep + Py groups. (C) Kaplan-Meier survival curves of mice in the Hep and Hep + Py groups. (D) Representative images of metastatic nodules in the abdominal cavity 15 days post-tumour cell inoculation. (E) Representative images of HCC nodules in the liver. Scale bar, $1 \mathrm{~cm}$. (F) The tumour volume in the Hep and Hep + Py groups was measured 15 days post-tumour cell inoculation. $(\mathrm{G})$ The tumour/body weight ratio in the Hep and Hep + Py groups measured 15 days post-tumour cell inoculation. $(\mathrm{H})$ The tumour/liver weight ratio in the Hep and Hep + Py groups measured 15 days post-tumour cell inoculation. $\mathrm{n}=26$. Data are presented as the mean \pm SD. ${ }^{* * *} \mathrm{P}<0.001$. Hep, mice inoculated with Hepa1-6 cells; Py, Plasmodium yoelii 17XNL; HCC, hepatocellular carcinoma.

$\left(0.23 \pm 0.05 \mathrm{~cm}^{3}\right)$ was significantly lower compared with that in the Hep group $\left(1.85 \pm 0.7 \mathrm{~cm}^{3}\right)$ (Fig. $1 \mathrm{E}$ and $\left.\mathrm{F}\right)$. The tumour weight on day 15 post-inoculation in the Hep + Py group was also significantly lower compared with that in the Hep group (Fig. 1G and H). In addition, only $2 / 15$ of mice were observed to have tumour metastasis in the intraperitoneal organs of the Hep + Py group, whereas severe intestinal and abdominal metastasis were present in 14/15 of mice in the Hep group (Fig. 1D).

Plasmodium infection suppresses HCC recurrence in a resection mouse model. The resection mouse model was established by intrahepatic implantation of tumour pieces, followed by complete tumour resection on day 21 post-implantation (Fig. 2A). In the early stage, no significant differences were observed in the body weight between the mice in the two groups.
Following tumour resection, weight loss and short-term weight recovery occurred in the Hep + Py group. During the period of infection, the food intake of mice in the Plasmodium infection group was poor; thus, the weight gain in the Plasmodium infection group was slower compared with that in the control group. Following self-healing from infection, the weight in the Plasmodium infection group continued to increase, whereas that of the control group decreased due to tumour development (Fig. 2B). The effect of surgical resection followed by Plasmodium infection on weight loss was notable when comparing the infected group in the resection model with the infected group in the non-resection model (Figs. 1B and 2B). A phenomenon of hemozoin accumulation (dark colour) (28) was observed in the liver in the Plasmodium-infected group in the resection (Fig. 2D) and the non-resection (Fig. 1E) models. Notably, the results demonstrated that the Plasmodium-treated 
A
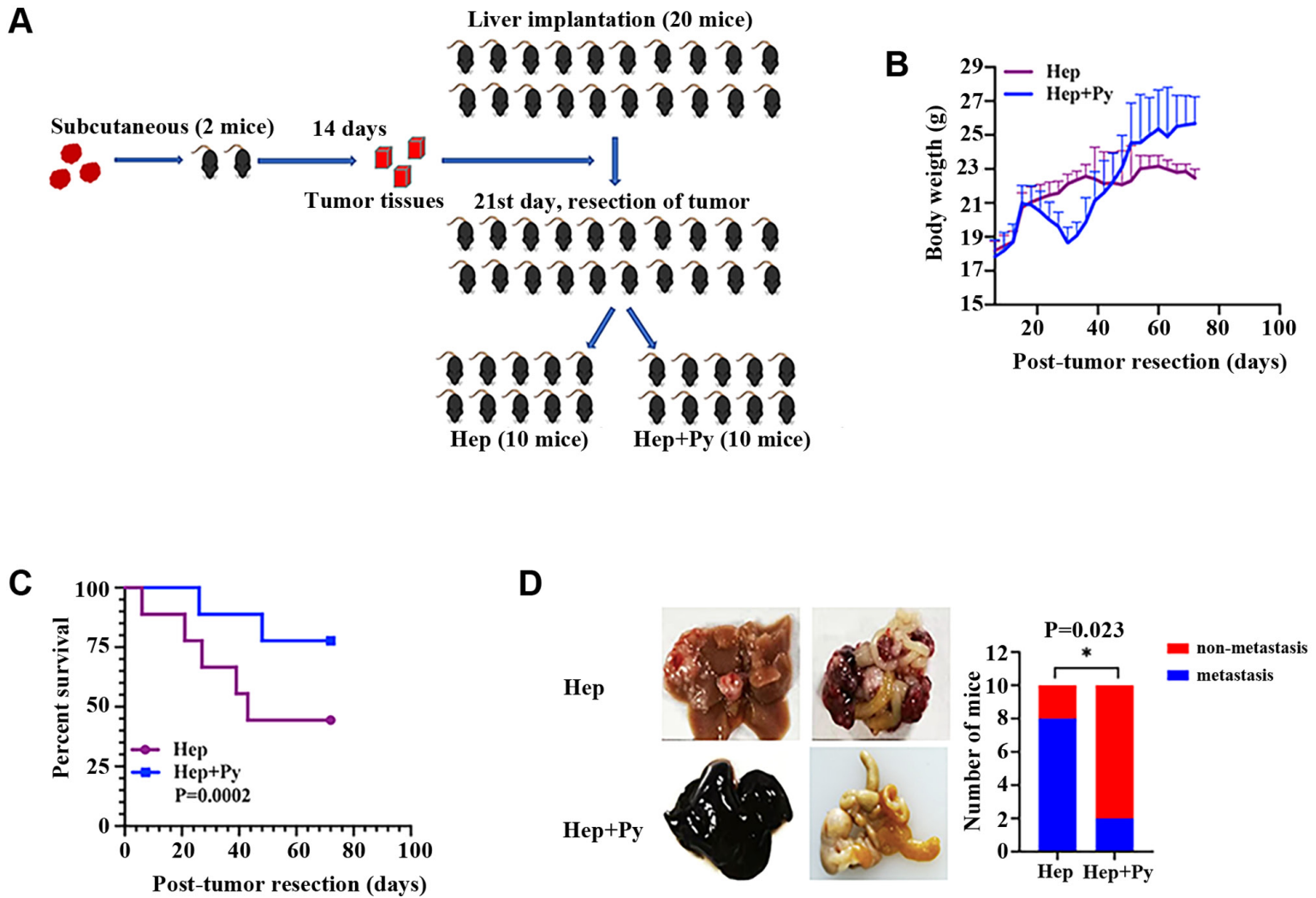

Figure 2. Plasmodium infection suppresses hepatocellular carcinoma progression and recurrence in the resection mouse model. (A) According to the experimental route, the tumour blocks of subcutaneous tumour-bearing mice were successfully removed and transplanted into the liver of experimental mice, and further grouped as Hep and Hep + Py for the next experiment. (B) Body weight curves of mice in the Hep and Hep + Py groups. (C) Kaplan-Meier survival curves of mice in the Hep and Hep + Py groups. (D) Representative images of metastatic nodules in the abdominal cavity and comparison of tumour metastasis between the two groups. ${ }^{*} \mathrm{P}<0.05$. Hep, mice inoculated with Hepa1-6 cells; Py, Plasmodium yoelii 17XNL.

tumour-bearing mice survived longer compared with the control mice (Fig. 2C). The cumulative recurrence rate in the Plasmodium infection group $(20 \%, 2 / 10)$ was significantly lower compared with that in the control group $(80 \%, 8 / 10)$ on day 75 post-inoculation (Fig. 2D).

Plasmodium infection inhibits HCC metastasis possibly through EMT suppression. E-cadherin is the key cadherin serves an antimetastatic role and is the most important EMT biomarker (6). In the present study, the expression levels of E-cadherin in the Hep + Py group were significantly higher compared with those in the Hep group (Fig. 3A). As one of the biomolecules that binds to the E-boxes in the E-cadherin promoter, Snail directly downregulates the expression of E-cadherin to promote the occurrence of EMT (29). The results of the present study demonstrated that the expression levels of Snail were significantly lower in the Hep + Py group compared with those in the Hep group (Fig. 3A). The RT-qPCR results showed that Vimentin expression, one of the molecular markers of EMT, was significantly decreased in the Hep + Py group compared with in the Hep group (Fig. 3B).

Plasmodium infection suppresses the activation of the PI3K/Akt/GSK-3 $\beta$ signalling pathway. Previous studies have reported that the PI3K/Akt/GSK-3 $\beta$ signalling pathway and the transcriptional repressor Snail are required for EMT in HCC $(14,16)$. Therefore, the effects of Plasmodium infection on the PI3K/Akt/GSK-3 $\beta$ signalling pathway was tested in HCC cells. Western blot analysis demonstrated that the phosphorylation of Akt (Ser473) (Fig. 4A and C) and GSK-3 3 (Ser9) (Fig. 4A and D) was downregulated in the Plasmodium-treated group compared with that in the control group, although the difference in PI3K phosphorylation levels between the two groups was not statistically significant (Fig. 4B).

Plasmodium infection downregulates the expression of CCR10. The chemokine/chemokine receptor axis has been demonstrated to activate the PI3K/Akt/GSK-3 $\beta /$ Snail signalling pathway in HCC cells $(14,16)$. As expected, the mRNA expression levels of CCR 10 were significantly lower in the Plasmodium-treated group compared with those in the control group (Fig. 5B). Western blotting results revealed that the protein expression levels of CCR10 were also significantly lower in the infected group compared with those in the control group (Fig. 5A).

\section{Discussion}

Primary HCC accounts for $75-85 \%$ of liver cancer (8.2\% of mortality rates in 2018), which is the fourth most common cause of cancer-related death worldwide $(2,30)$. Recurrence is the leading cause of postoperative death in patients with liver cancer (31). The results of the present study demonstrated that Plasmodium infection significantly inhibited tumour growth 
A
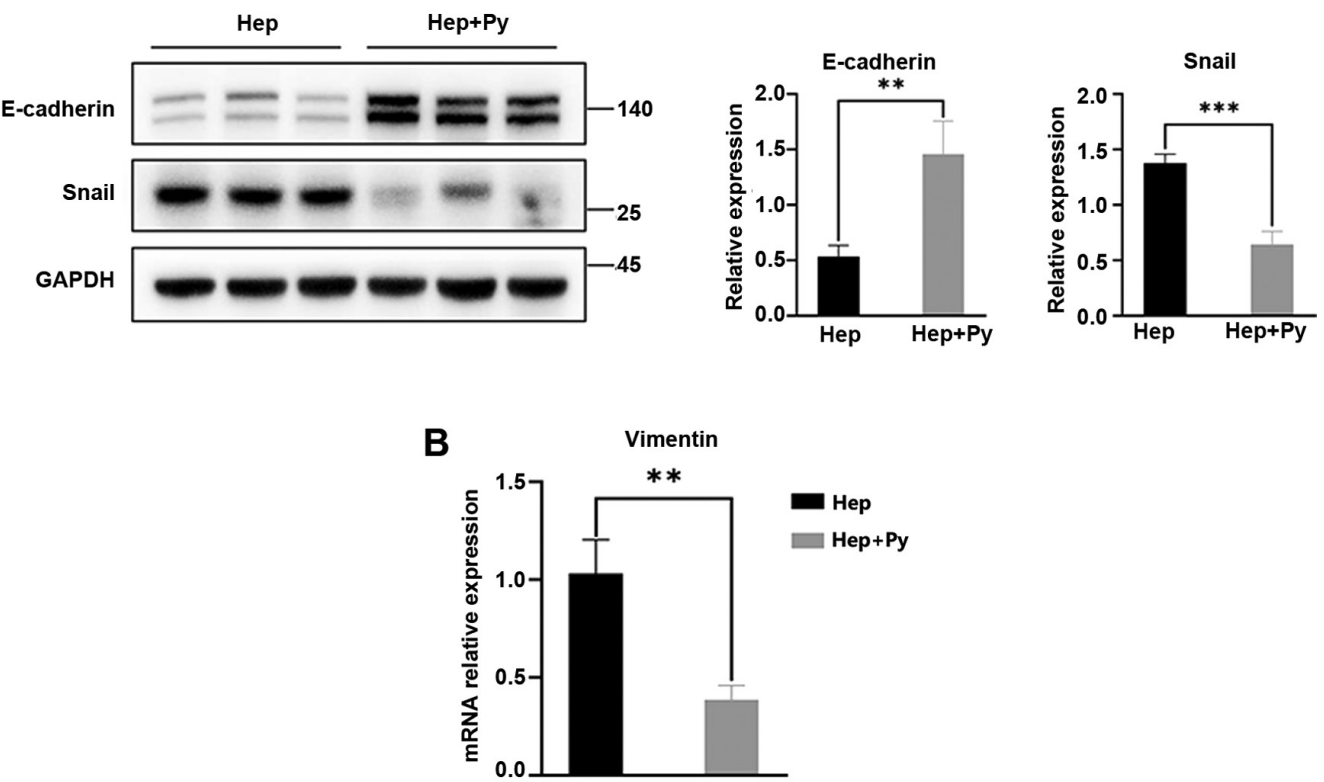

Figure 3. Plasmodium infection downregulates the expression levels of Snail and Vimentin and upregulates the expression levels of E-cadherin. (A) Western blot analysis of the levels of E-cadherin and Snail in tumour tissues of the Hep and Hep + Py. (B) Reverse transcription-quantitative PCR results of Vimentin expression showed that the level of mRNA in Hep + Py was significantly decreased compared with in the Hep group. Data are presented as the mean \pm SD ${ }^{* *} \mathrm{P}<0.01,{ }^{* * *} \mathrm{P}<0.001$. Hep, mice inoculated with Hepa1-6 cells; Py, Plasmodium yoelii 17XNL.

A

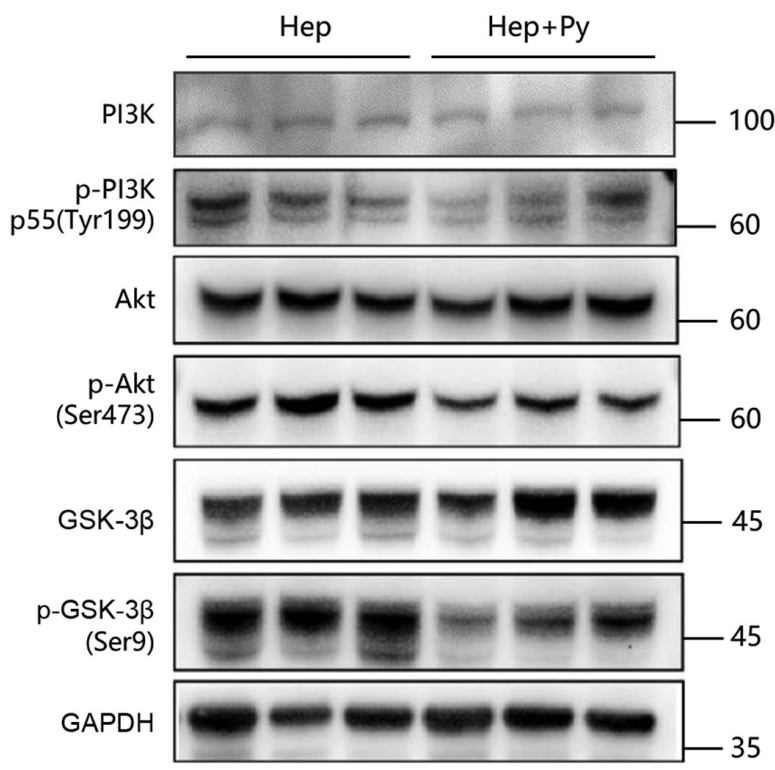

B

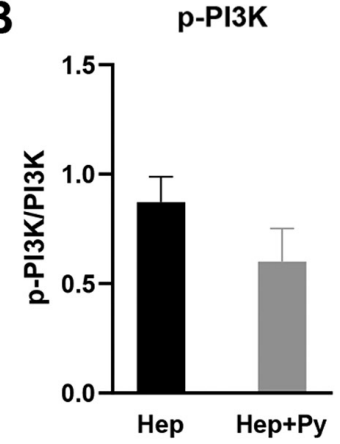

C

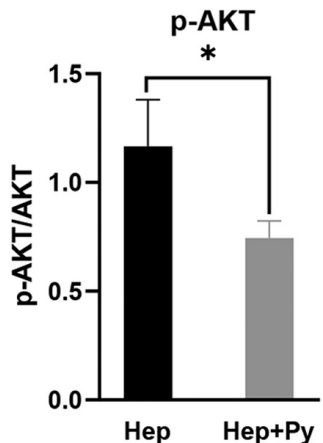

D

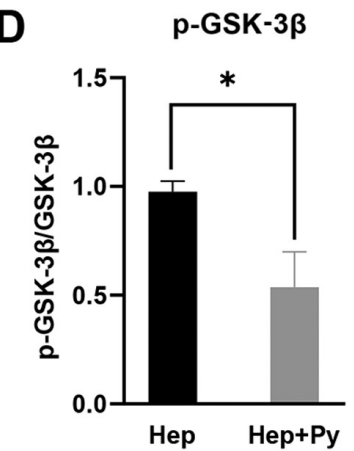

Figure 4. Plasmodium infection suppresses the activation of the PI3K/Akt/GSK-3 $\beta$ signalling pathway. (A) Western blot analysis of PI3K, p-PI3K, Akt, p-Akt,

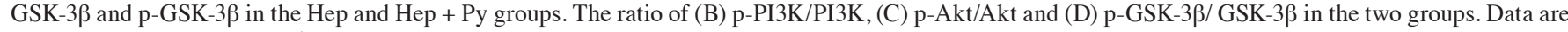
presented as the mean \pm SD. "P<0.05. Hep, mice inoculated with Hepa1-6 cells; Py, Plasmodium yoelii 17XNL; p-, phosphorylated. 
A

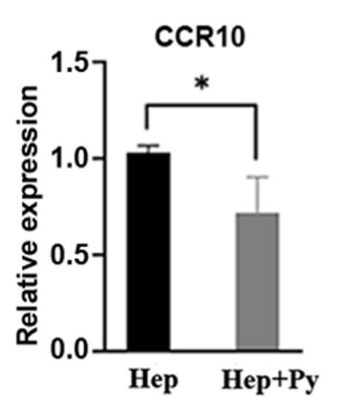

B CCR10

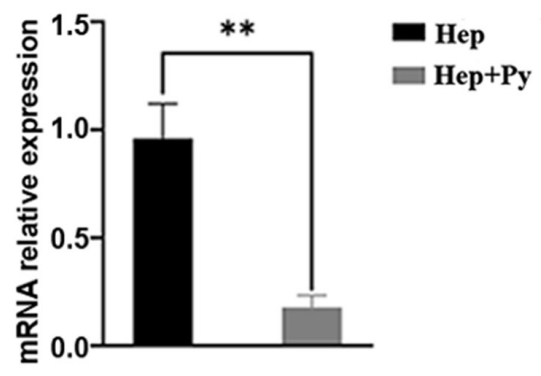

Figure 5. Plasmodium infection downregulates the expression of CCR10. (A) Western blot analysis of CCR10. (B) The mRNA expression levels of CCR10. Data are presented as the mean \pm SD. ${ }^{*} \mathrm{P}<0.05$ and ${ }^{* *} \mathrm{P}<0.01$. CCR10, CC-chemokine receptor 10; Hep, mice inoculated with Hepa1-6 cells; Py, Plasmodium yoelii $17 \mathrm{XNL}$.

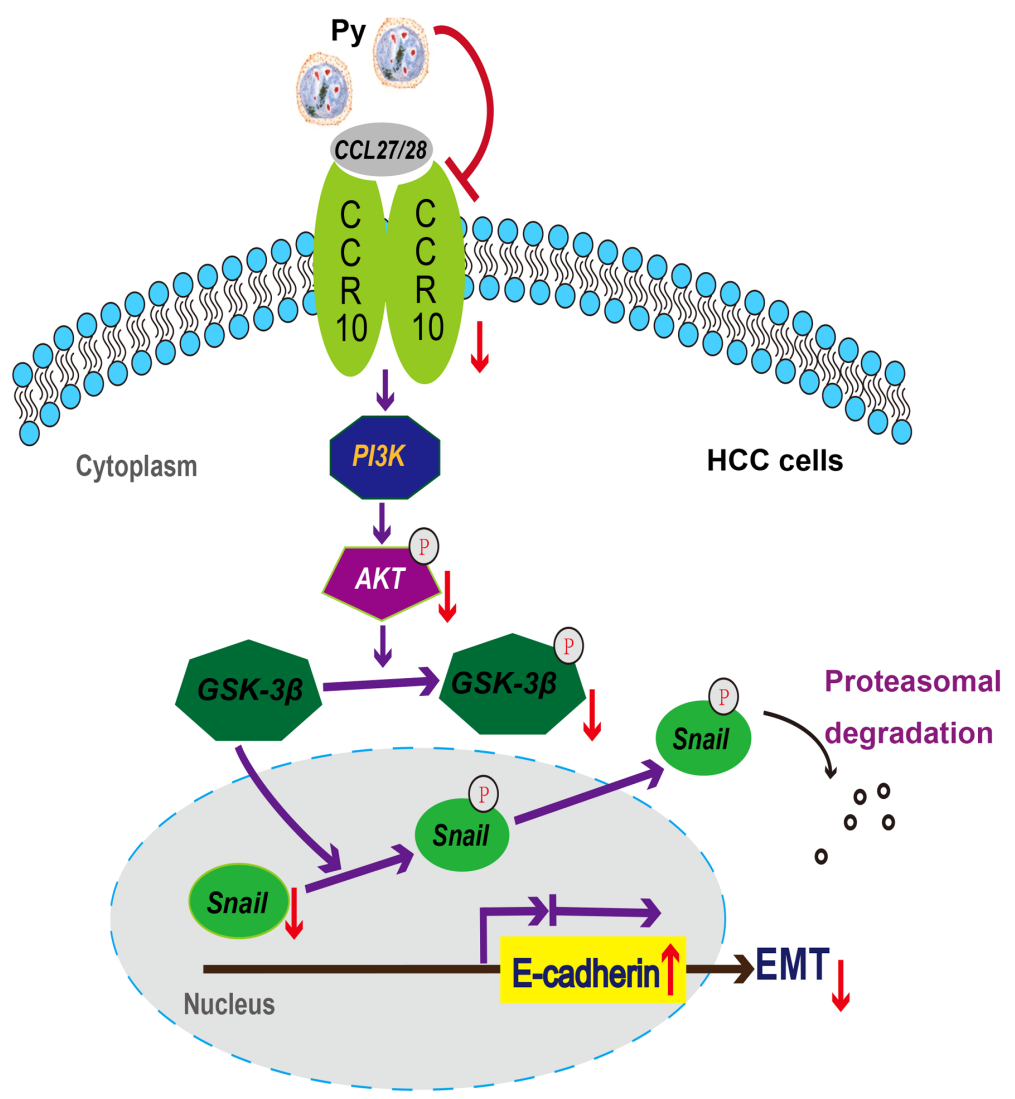

Figure 6. Potential mechanism of Plasmodium infection against recurrence and metastasis of HCC in mice. Plasmodium infection induces: i) The down-

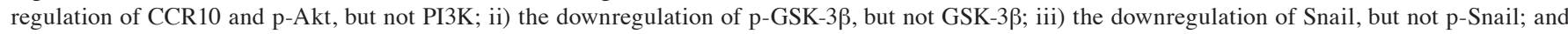
iv) the upregulation of E-cadherin. Notably, the downregulation of Snail and the upregulation of E-cadherin may contribute to the suppression of the EMT in murine HCC resection and non-resection models. HCC, hepatocellular carcinoma; CCR10, CC-chemokine receptor 10; Py, Plasmodium yoelii 17XNL; p-, phosphorylated; EMT, epithelial-mesenchymal transition; CCL, C-C motif chemokine ligand.

and abdominal metastasis in two mouse models, including a murine HCC resection model. The underlying mechanisms of action may involve the inhibition of the PI3K/Akt/GSK-3 $\beta /$ Snail signalling pathway by downregulating the expression levels of 
CCR10, reducing the accumulation of Snail and upregulating the levels of E-cadherin.

The EMT has been implicated in carcinoma invasion and metastasis (32). Previous studies have suggested that the downregulation of E-cadherin is a prominent feature of the EMT. For example, the transcriptional activity and protein expression of E-cadherin has been reported to be enhanced by preventing the binding of the E-boxes of the E-cadherin promoter to transcriptional barriers, including zinc-finger transcriptional repressors snail and slug, the repressor zinc-finger E-box-binding homeobox (ZEB)-2, ZEB-1, as well as the basic helix-loop-helix, transcription factors twist and E12/E47, which prevent the EMT $(32,33)$. The results of the current study demonstrated that Plasmodium infection increased the expression levels of E-cadherin and downregulated the levels of Snail and Vimentin compared with those in the control group, which suggested that Plasmodium infection may prevent the EMT in HCC.

PI3K activates the secondary messenger PIP3, which activates Akt via phosphorylation at Ser308 (34). GSK-3 $\beta$ is a downstream target of p-Akt (35), which promotes the degradation of Snail (36), whereas the phosphorylation of GSK-3 $\beta$ at Ser9 by PI3K/Akt leads to its inactivation (37) and upregulation of Snail. Snail, which downregulates E-cadherin, is downstream to GSK-3 $\beta$; GSK-3 $\beta$ inhibits the expression of Snail and increases the expression levels of E-cadherin (38). The results of the current study demonstrated that Plasmodium infection inhibited the phosphorylation of Akt, which subsequently inhibited the conversion of GSK-3 $\beta$ to $\mathrm{p}-\mathrm{GSK}-3 \beta$ and reduced the expression levels of Snail. Therefore, the inhibitory effects of Snail on E-cadherin expression were eliminated, and the EMT was prevented.

Chemokines exert their action through seven transmembrane spanning G-protein-linked chemokine receptors $(39,40)$. Previous studies have suggested that chemokines and chemokine receptors are involved in inflammatory reactions and wound healing (41-43). A recent study has demonstrated that chemokines and their receptors serve a crucial role in tumour cell growth and metastasis in melanoma, lung cancer, gastric carcinoma, pancreatic cancer, colorectal carcinoma and HCC (44). CCR10 activation has been reported to stimulate the invasion and migration of HCC, breast cancer and melanoma cells $(16,45,46)$. CCR10 and the downstream PI3K/Akt signalling pathway serve a role in HCC progression (16). The results of the present study demonstrated that Plasmodium infection downregulated the expression levels of CCR10 compared with those in the control group. The inhibition of CCR10-mediated $\mathrm{PI} 3 \mathrm{~K} / \mathrm{Akt} / \mathrm{GSK}-3 \beta /$ Snail signalling may result in the suppression of the EMT programming in HCC. However, one limitation of the present study was that experiments were only repeated twice.

In conclusion, the results of the current study identified a potential novel mechanism of Plasmodium infection against HCC in mice (Fig. 6), providing evidence for the prevention of recurrence and distant metastasis of $\mathrm{HCC}$ by regulating the EMT through infection with Plasmodium parasites. In addition, our previous studies have demonstrated that Plasmodium infection displays antitumor effects by activating the innate and acquired antitumor immunity, remodelling the tumour immunosuppressive microenvironment and inhibiting angiogenesis (20-25). Based on these studies, three clinical trials are ongoing in China (trial nos. NCT02786589, NCT03474822 and NCT04165590). These findings may be important for developing novel strategies for the treatment and prevention of metastasis and recurrence of $\mathrm{HCC}$, especially for patients in the perioperative period.

\section{Acknowledgements}

The authors would like to thank Dr Zhenhui Li (The Third Affiliated Hospital of Kunming Medical University, Yunnan Cancer Hospital, Yunnan Cancer Center, Kunming, China) and Dr Wei Guo (Guangzhou University of Chinese Medicine, Guangzhou, China) for their assistance in the animal experiments.

\section{Funding}

The present study was supported by the Key Regional Project of Science and Technology Service Network Program of The Chinese Academy of Sciences (grant no.KFJ-STS-QYZX-042), the Science and Technology Program of Guangzhou, China (grant no. 201707010447), The National Natural Science Foundation of China (grant no. 81673003), the Clinical Research Program of High Level University of Guangzhou Medical University 2017, the State Key Laboratory of Respiratory Diseases (grant no. SKLRD-OP-201802) and the Applied Basic Research Projects of Yunnan Province, China (grant no. 2019FE001-054).

\section{Availability of data and materials}

All data generated or analysed during this study are included in this published article. The manuscript has been published as a preprint.

\section{Authors' contributions}

XPC, XWZ, YL, XC and LQ conceived and designed the study. YL, XC, MM and LQ completed in vitro culture experiments and performed the in vivo operations. ZT, WTD and LLD assisted in the in vivo operations and conducted the staining experiments. YL, MM, DA and SF carried out the western blotting and PCR assays. YL, MM, XC, STZ and XFL performed the data analysis and interpretation. XFL, XWZ, XPC and LQ made substantial contributions to the writing of the manuscript. All authors agreed to be accountable for all aspects of the work. YL, XC, XPC and XWZ confirmed the authenticity of all the raw data. All authors read and approved the final manuscript.

\section{Ethics approval and consent to participate}

The specific procedures used during the animal study were approved by the Ethics Committee of The Animal Laboratory Center of Guangzhou Institutes of Biomedicine and Health, Chinese Academy of Sciences, and obtained the corresponding IACUC (approval no. N2019014). All efforts were made to minimise animal suffering. 


\section{Patient consent for publication}

Not applicable.

\section{Competing interests}

The authors declare that they have no competing interests.

\section{References}

1. Global Burden of Disease Liver Cancer Collaboration, Akinyemiju T, Abera S, Ahmed M, Alam N, Alemayohu MA, Allen C, Al-Raddadi R, Alvis-Guzman N, Amoako Y, et al: The burden of primary liver cancer and underlying etiologies from 1990 to 2015 at the global, regional, and national level: Results from the global burden of disease study 2015. JAMA Oncol 3 : 1683-1691, 2017

2. Bray F, Ferlay J, Soerjomataram I, Siegel RL, Torre LA and Jemal A: Global cancer statistics 2018: GLOBOCAN estimates of incidence and mortality worldwide for 36 cancers in 185 countries. CA Cancer J Clin 68: 394-424, 2018.

3. Torre LA, Bray F, Siegel RL, Ferlay J, Lortet-Tieulent J and Jemal A: Global cancer statistics, 2012. CA Cancer J Clin 65: 87-108, 2015

4. Sharma SA, Kowgier M, Hansen BE, Brouwer WP, Maan R, Wong D, Shah H, Khalili K, Yim C, Heathcote EJ, et al: Toronto HCC risk index: A validated scoring system to predict 10 -year risk of HCC in patients with cirrhosis. J Hepatol, Aug 24, 2017 (Online ahead of print)

5. Zhou L, Rui JA, Wang SB, Chen SG and Qu Q: Risk factors of microvascular invasion, portal vein tumor thrombosis and poor post-resectional survival in HBV-related hepatocellular carcinoma. Hepatogastroenterology 61: 1696-1703, 2014.

6. Giannelli G, Koudelkova P, Dituri F and Mikulits W: Role of epithelial to mesenchymal transition in hepatocellular carcinoma. J Hepatol 65: 798-808, 2016.

7. Thiery JP and Sleeman JP: Complex networks orchestrate epithelial-mesenchymal transitions. Nat Rev Mol Cell Biol 7: 131-142, 2006

8. Wang Y, Shi J, Chai K, Ying X and Zhou BP: The role of snail in EMT and tumorigenesis. Curr Cancer Drug Targets 13: 963-972, 2013.

9. Kaufhold S and Bonavida B: Central role of Snaill in the regulation of EMT and resistance in cancer: A target for therapeutic intervention. J Exp Clin Cancer Res 33: 62, 2014.

10. Yuan K, Xie K, Lan T, Xu L, Chen X, Li X, Liao M, Li J, Huang J, Zeng Y and Wu H: TXNDC12 promotes EMT and metastasis of hepatocellular carcinoma cells via activation of $\beta$-catenin. Cell Death Differ 27: 1355-1368, 2020.

11. Ma M, Xu H, Liu G, Wu J, Li C, Wang X, Zhang S, Xu H, Ju S, Cheng W, et al: Metabolism-induced tumor activator 1 (MITA1), an energy stress-inducible long noncoding RNA, promotes hepatocellular carcinoma metastasis. Hepatology 70: 215-230, 2019.

12. Peng JM, Bera R, Chiou CY, Yu MC, Chen TC, Chen CW, Wang TR, Chiang WL, Chai SP, Wei Y, et al: Actin cytoskeleton remodeling drives epithelial-mesenchymal transition for hepatoma invasion and metastasis in mice. Hepatology 67: 2226-2243, 2018.

13. Wan S, Meyer AS, Weiler SME, Rupp C, Tóth M, Sticht C, Singer S, Thomann S, Roessler S, Schorpp-Kistner M, et al: Cytoplasmic localization of the cell polarity factor scribble supports liver tumor formation and tumor cell invasiveness. Hepatology 67: 1842-1856, 2018.

14. Zhou SL, Zhou ZJ, Hu ZQ, Li X, Huang XW, Wang Z, Fan J, Dai $Z$ and Zhou J: CXCR2/CXCL5 axis contributes to epithelial-mesenchymal transition of HCC cells through activating PI3K/Akt/GSK-3ß/Snail signaling. Cancer Lett 358: 124-135, 2015.

15. Cui D,Zhao Y and Xu J: Activated CXCL5-CXCR2 axis promotes the migration, invasion and EMT of papillary thyroid carcinoma cells via modulation of $\beta$-catenin pathway. Biochimie 148: $1-11$, 2018.

16. Wu Q, Chen JX, Chen Y, Cai LL, Wang XZ, Guo WH and Zheng JF: The chemokine receptor CCR10 promotes inflammation-driven hepatocarcinogenesis via PI3K/Akt pathway activation. Cell Death Dis 9: 232, 2018.
17. Tavares J, Formaglio P, Thiberge S, Mordelet E, Van Rooijen N Medvinsky A, Ménard R and Amino R: Role of host cell traversal by the malaria sporozoite during liver infection. J Exp Med 210: 905-915, 2013.

18. Odedra A, Webb L, Marquart L, Britton LJ, Chalon S, Moehrle JJ, Anstey NM, William T, Grigg MJ, Lalloo DG, et al: Liver function test abnormalities in experimental and clinical Plasmodium vivax infection. Am J Trop Med Hyg 103: 1910-1917, 2020.

19. Qin L, Chen C, Chen L, Xue R, Ou-Yang M, Zhou C, Zhao S, $\mathrm{He} \mathrm{Z}$, Xia Y, He J, et al: Worldwide malaria incidence and cancer mortality are inversely associated. Infect Agent Cancer 12: 14, 2017.

20. Adah D, Yang Y, Liu Q, Gadidasu K, Tao Z, Yu S, Dai L, Li X, Zhao S, Qin L, et al: Plasmodium infection inhibits the expansion and activation of MDSCs and Tregs in the tumor microenvironment in a murine Lewis lung cancer model. Cell Commun Signal 17: 32, 2019.

21. Shi X, Qin L, Liu G, Zhao S, Peng N and Chen X: Dynamic balance of pSTAT1 and pSTAT3 in C57BL/6 mice infected with lethal or nonlethal Plasmodium yoelii. Cell Mol Immunol 5: 341-348, 2008.

22. Chen L, He Z, Qin L, Li Q, Shi X, Zhao S, Chen L, Zhong N and Chen X: Antitumor effect of malaria parasite infection in a murine Lewis lung cancer model through induction of innate and adaptive immunity. PLoS One 6: e24407, 2011.

23. Liu Q, Yang Y, Tan X, Tao Z, Adah D, Yu S, Lu J, Zhao S, Qin L, Qin L and Chen X: Plasmodium parasite as an effective hepatocellular carcinoma antigen glypican-3 delivery vector. Oncotarget 8: 24785-24796, 2017.

24. Yang Y, Liu Q, Lu J, Adah D, Yu S, Zhao S, Yao Y, Qin L, Qin $\mathrm{L}$ and Chen X: Exosomes from Plasmodium-infected hosts inhibit tumor angiogenesis in a murine Lewis lung cancer model. Oncogenesis 6: e351, 2017.

25. Wang B, Li Q, Wang J, Zhao S, Nashun B, Qin L and Chen X: Plasmodium infection inhibits tumor angiogenesis through effects on tumor-associated macrophages in a murine implanted hepatoma model. Cell Commun Signal 18: 157, 2020.

26. Livak KJ and Schmittgen TD: Analysis of relative gene expression data using real-time quantitative PCR and the 2(-Delta Delta C(T)) method. Methods 25: 402-408, 2001.

27. R Core Team: R: A language and environment for statistical computing. R Foundation for Statistical Computing, Vienna, 2014.

28. Levesque MA, Sullivan AD and Meshnick SR: Splenic and hepatic hemozoin in mice after malaria parasite clearance. J Parasitol 85: 570-573, 1999.

29. Barrallo-Gimeno A and Nieto MA: The Snail genes as inducers of cell movement and survival: Implications in development and cancer. Development 132: 3151-3161, 2005.

30. Villanueva A: Hepatocellular carcinoma. N Engl J Med 380: $1450-1462,2019$

31. Buonaguro L, Mauriello A, Cavalluzzo B, Petrizzo A and Tagliamonte M: Immunotherapy in hepatocellular carcinoma. Ann Hepatol 18: 291-297, 2019.

32. Yang $\mathbf{J}$ and Weinberg RA: Epithelial-mesenchymal transition: At the crossroads of development and tumor metastasis. Dev Cell 14: 818-829, 2008.

33. Eger A, Aigner K, Sonderegger S, Dampier B, Oehler S, Schreiber M, Berx G, Cano A, Beug H and Foisner R: DeltaEF1 is a transcriptional repressor of E-cadherin and regulates epithelial plasticity in breast cancer cells. Oncogene 24: 2375-2385, 2005.

34. Elich M and Sauer K: Regulation of hematopoietic cell development and function through phosphoinositides. Front Immunol 9: 931,2018

35. Zhang C, Wei S, Sun WP, Teng K, Dai MM, Wang FW, Chen JW, Ling H, Ma XD, Feng ZH, et al: Super-enhancer-driven AJUBA is activated by TCF4 and involved in epithelial-mesenchymal transition in the progression of hepatocellular carcinoma. Theranostics 10: 9066-9082, 2020

36. Lee YJ and Han HJ: Troglitazone ameliorates high glucoseinduced EMT and dysfunction of SGLTs through PI3K/Akt, GSK-3 $\beta$, Snail1, and $\beta$-catenin in renal proximal tubule cells. Am J Physiol Renal Physiol 298: F1263-F1275, 2010.

37. Liang Y, Jing Z, Deng H, Li Z, Zhuang Z, Wang S and Wang Y: Soluble epoxide hydrolase inhibition ameliorates proteinuria-induced epithelial-mesenchymal transition by regulating the PI3K-Akt-GSK-3 $\beta$ signaling pathway. Biochem Biophys Res Commun 463: 70-75, 2015. 
38. Zhou BP, Deng J, Xia W, Xu J, Li YM, Gunduz M and Hung MC: Dual regulation of Snail by GSK-3beta-mediated phosphorylation in control of epithelial-mesenchymal transition. Nat Cell Biol 6: 931-940, 2004

39. Amarandi RM, Hjortø GM, Rosenkilde MM and Karlshøj S: Probing biased signaling in chemokine receptors. Methods Enzymol 570: 155-186, 2016.

40. Zweemer AJM, Toraskar J, Heitman LH and Ijzerman AP: Bias in chemokine receptor signalling. Trends Immunol 35: 243-252, 2014.

41. Roh YS and Seki E: Chemokines and chemokine receptors in the development of NAFLD. Adv Exp Med Biol 1061: 45-53, 2018

42. Ridiandries A, Tan JTM and Bursill CA: The role of chemokines in wound healing. Int J Mol Sci 19: 3217, 2018

43. Kieseier BC, Tani M, Mahad D, Oka N, Ho T, Woodroofe N, Griffin JW, Toyka KV, Ransohoff RM and Hartung HP: Chemokines and chemokine receptors in inflammatory demyelinating neuropathies: A central role for IP-10. Brain 125: 823-834, 2002
44. Marcuzzi E, Angioni R, Molon B and Calì B: Chemokines and chemokine receptors: Orchestrating tumor metastasization. Int J Mol Sci 20: 96, 2018.

45. Lin HY, Sun SM, Lu XF, Chen PY, Chen CF, Liang WQ and Peng CY: CCR 10 activation stimulates the invasion and migration of breast cancer cells through the ERK1/2/MMP-7 signaling pathway. Int Immunopharmacol 51: 124-130, 2017.

46. Monteagudo C, Ramos D, Pellín-Carcelén A, Gil R, Callaghan RC, Martín JM, Alonso V, Murgui A, Navarro L, Calabuig S, et al: CCL27-CCR10 and CXCL12-CXCR4 chemokine ligand-receptor mRNA expression ratio: New predictive factors of tumor progression in cutaneous malignant melanoma. Clin Exp Metastasis 29: 625-637, 2012.

This work is licensed under a Creative Commons Attribution-NonCommercial-NoDerivatives 4.0 International (CC BY-NC-ND 4.0) License. 